I $\mathrm{H}-\left(300 \mathrm{MHz}, \mathrm{CDCl}_{3}\right)$ und ${ }^{13} \mathrm{C}-\mathrm{NMR}\left(75 \mathrm{MHz}, \mathrm{CDCl}_{3}\right)$ : vgl. Tabelle.

Generierung und Abfangen von Tricyclo[3.2.1.02,4]octa-2(4),6-dien (12). In einem ausgeheizten 25-ml-Dreihals-Rundkolben mit Septum, Thermometer und Inertgas-Überleitung werden 200 $\mathrm{mg}(0,76 \mathrm{mmol}) \mathbf{1 5} \mathrm{a}$ und $205 \mathrm{mg}(0,76 \mathrm{mmol})$ DPIBF in $5 \mathrm{ml} \mathrm{THF}$ gelöst und während 15 min mittels Spritze bei $-80^{\circ}$ bis $-75^{\circ} 0,53 \mathrm{ml}$ einer $1,4 \mathrm{M} \quad t$-BuLi Lsg. in Pentan $(0,74 \mathrm{mmol})$ zugetropft. Nach jedem Tropfen verfärbt sich die gelbe Lsg. braun. Die gelbbraune Lsg. wird während $30 \mathrm{~min}$ bei $-50^{\circ}$, dann $1 \mathrm{~h}$ bei RT. gerührt. Zur Elimination von evtl, überschüssigem DPIBF wird mit ca. $200 \mathrm{mg}$ (ca. 2,0 mmol) Maleinsäure-anhydrid versetzt und $15 \mathrm{~min}$ weitergerührt. Das Gemisch wird mil $50 \mathrm{ml} \mathrm{Et} 2 \mathrm{O}$ verdünnt, im Scheidetrichter $2 \times$ mit $2 \mathrm{~N}$ $\mathrm{NaOH}, 1 \times$ mit $\mathrm{H}_{2} \mathrm{O}$ und $\mathrm{I} x$ mit ges. $\mathrm{NaCl}$-Lsg. gewaschen, getrocknet $\left(\mathrm{MgSO}_{4}\right)$ und i.RV, bei RT. einrotiert. Das ölige Rohprodukt wird in 2 Teilen durch präp. Schichtchromatographie gereinigt (Merck Art 5717 PSC-Fertigplatten Kieselgel $60 F_{254}$, Schichtdicke: $2 \mathrm{~mm}$, Elutionsmittel Pentan/ $\mathrm{Et}_{2} \mathrm{O}$ 20:1, zweifache Elution). Die Zone mit einem $R_{\mathrm{f}}$-Wert von 0,61 wird gesammelt und mit $\mathrm{E}_{2} \mathrm{O}$ extrahiert, das Eluat i.RV bei RT. eingedampft: $119,8 \mathrm{mg}(42 \%)$ weisse Kristalle von 16, die laut lH-NMR nur Spuren von Verunrcinigungen enthalten. Schmp. $172-173^{\circ}$ (Pentan). ${ }^{1} \mathrm{H}-$ $\left(300 \mathrm{MHz}, \mathrm{CDCl}_{3}\right)$ und ${ }^{13} \mathrm{C}-\mathrm{NMR}\left(75 \mathrm{MHz}, \mathrm{CDCl}_{3}\right)$ : vgl. Tabelle. MS: $374\left(81, M^{+}\right), 307(100)$

2-Bromo-4-(trimethylsilyl)-endo-tricyclo[3.2.1.02.4 ]oci-6-en (15b). In einem ausgeheizten 25ml-Dreihals-Rundkolben mit Septum, Thermometer und Inertgas-Überleitung wird eine Lsg. von $500 \mathrm{mg}$ (1.42 mmol) 1,1,2-Tribromo-2-(trimethylsilyl)cyclopropan (13) [ 10 ] in $5 \mathrm{ml} \mathrm{THF}$ auf $-80^{\circ}$ abgekühlt. Man tropft mittels Spritze während $15 \mathrm{~min} 1,01 \mathrm{ml}$ einer 1,4M /-BuLi Lsg. in Pentan (1,4l mmol) zu. Dabei tritt eine deutliche Farbveränderung von violett über grüngelb bis braun ein. Innerhalb von $30 \mathrm{~min}$ wird unter Rühren von $-80^{\circ}$ auf $-50^{\circ}$, sodann innert $10 \mathrm{~min}$ von $-50^{\circ}$ auf RT. erwirmt und rasch mit ciner Lsg. von $0,116 \mathrm{ml}(93 \mathrm{mg}, 1,41 \mathrm{mmol})$ Cyclopentadien in $\mathrm{I} \mathrm{ml}$
THF versetzt. Das braune Gemisch wird weitere $4,5 \mathrm{~h}$ bei RT. gerührt, mit $50 \mathrm{ml} \mathrm{Et}_{2} \mathrm{O}$ verdünnt, im Scheidetrichter mit $\mathrm{H}_{2} \mathrm{O}$ und ges. $\mathrm{NaCl}-\mathrm{Lsg}$. gewaschen, getrocknet $\left(\mathrm{MgSO}_{4}\right)$ und i. RV. bei $-20^{\circ} / 0,2$ Torr eingeengt: $253,7 \mathrm{mg}$ (69\%) NMR-reines Rohprodukt $15 \mathrm{~b}$ als gelbliches Öl. Chromatographie über $15 \mathrm{~g}$ Kieselgel mit Pentan liefert $166,8 \mathrm{mg}(46 \%)$ 15b als farblose Flüssigkeit. ${ }^{1} \mathrm{H}-\left(300 \mathrm{MHz}, \mathrm{CDCl}_{3}\right)$ und ${ }^{13} \mathrm{C}$ NMR (75 $\left.\mathrm{MHz}_{2} \mathrm{CDCl}_{3}\right)$ : vgl. Tabelle.

\section{Eingegangen am 30 . November 1990}

[1] Teil der geplanten Dissertation von M.M., Universität Bern.

[2] W.E. Billups, M.M. Haley, G. A. Lee, Chem, Rev $1989,89,1147$; B. Halton, M.G. Banwell, 'Cyclopropenes', in 'The Chemistry of the Cyclopropyl Group', Ed. Z. Rappoport, Wiley, New York, 1987

[3] H.-U. Wagner, G. Szeimies, J. Chandrasekhar, P.v.R. Schleyer, J.A. Pople, J.S. Binkley, J. Am. Chem. Soc. 1978, 100, 1210.

[4] G. Szeimies, J. Harnisch, 0. Baumgärtel, J. Am. Chem. Soc. 1977, 99, 5183; U. Szeinies-Seebach, G. Szeimies, ihid. 1978, 100, 3966; U. Szeimies-Seebach, A. Schöffer, R. Römer, G. Szeimies, Chem. Ber. 1981, 114, 1767; H.-G. Zoch, G. Szeimies, R. Römer, G. Germain, J.-P. Declercq, ibid. 1983, //6, 2285.

[5] A. Weber, M. Neuenschwander, Angew. Chem. 1981, 93, 788; ibid. Int. Ed. 1981, 20, 774; A. Weber, U. Stämpfli, M. Neuenschwander, Helv. Chim. Acta 1989, 72, 29.

[6] W.E. Billups, L.-J. Lin, E.W. Casserley, J. Am. Chem. Soc. 1984, 106, 3698; S.W. Staley, T.D. Norden, ibid. 1984, 106, 3699.

[7] K.B. Wiberg, G. Bonneville, Tetrahedron Lett. $1982,23,5385$.

[8] W.E. Billups, L.-J . Lin, Tetrahedron 1986, 42 , 1575.

[9] P.J.Chenier, D.A. Southard, J.Org. Chem. 1989, 54, 3519; ibid. 1990, 55, 4333 .
[10] B.R. Dent, B. Halton, A.M.F. Smith. Aust, J. Chem. 1986, 39, 1621.

[11] In Abwesenheit von Cyclopentadien liisst sich 1,2-Dibromocyclopropen (14a) NMRspektroskopisch nachweisen: Das charakteristische Singulett der $\mathrm{CH}_{2}$-Gruppe bei $2,10 \mathrm{ppm}$ $\left(\mathrm{CDCl}_{3}\right)$ verschwindet bei Zugabe von Cyclopentadien.

[12] St.Walther, Dissertation, Universitit Bern, 1985.

[13] Während $C(8)$ von endo-Tricyclol.3.2.1.0 $0^{2.4} \mathrm{loct}-$ 6 -en bei $63.7 \mathrm{ppm}$ absorbiert. liegt $\mathrm{C}(8)$ des e'voIsomers bei $37,4 \mathrm{ppm}$ ! H. Günther, W. Herrig, $H$. Seel, S. Tobias, A de Meijere, B. Schrader, I.Org. Chen. 1980, 45, 4329; K. Tori, M. Ueyama, T. Tsuji, H. Matsumura, H. Tanida, H. I wamura, K. Kushida, T, Nishida, S. Satoh, Tetrahedron Le'll. 1974, 327.

[14] Die Abfangreaktion von 12 ist nur zu $40 \%$ effizient, die Struktur möglicher Nebenprodukte noch unbekannt. Als Folgereaktion von 12 ist vor allem dic Drciringöffnung zu einem Vinylcarben attraktiv, welches sich durch Umlagerung stabilisieren kinn. Vgl. z.B. P. Miiller, N. Pautex, Hels. Chim. Acta 1988, 71, 1630; N. Piutex, Dissertation, Universitiat Genf, 1989.

[15] Trotz dieser uberzeugenden Argumente soll versucht werden, die Struktur von 16 durch eine Röntgenstrukturanalyse zu bestïtigen.

[16] Sowohl die Signallage als auch die Kopplungskonstante $J(3 a, 3 b)$ von 16 sind den Werten analoger syn-konfigurierter Addukte von Diphenylisobenzofuran an Cyclopropene sehr ähnlich. Vgl. P. Müller, G. Bernardinelli, J. Pfyffer, D. Rodriguez, J.-P. Schaller, Holl, Chim. Acta 1988, 71, 544

[17] Aufgrund von Vorversuchen verläufı der (Halogen-Li)-Austausch sogar schneller als dic Deprotonierung von Cyclopentadien, so dass das Cyclopropan 13 mit $t$-BuLi sogar in Gegenwart von Cyclopentadien umgesetzi werden kann!

[18] W.E. Billups, M.M. Haley, Angen: Chem. 1989, 101, 1735; ibid. Int. Ed. 1989, 28, 1711.
Chimia 45 (1991) 27-29

(c) Schweiz. Chemiker-Verband; ISSN 0009-4293

\title{
Isolierung und Charakterisierung der Hauptcarotinoide aus Blütenblättern von Brassica napus
}

\section{Bertold Debrunner und Hanspeter Pfander*}

Abstract. From the yellow petals of rape (Brassica napus), five major carotenoids have been isolated. Based on UV/VIS, CD, 400-MHz' $\mathrm{H}-\mathrm{NMR}$, and mass spectra, they were identified as $\left(9^{\prime} Z\right)$-neoxanthin, (all-E)- and (9Z)-violaxanthin, and $\left(8^{\prime} R\right)$ - and ( $\left.8^{\prime} S\right)$-luteoxanthin.

\section{Einleitung}

Raps (Brassicanapus) ist im gemässigten Klimabereich der nördlichen Hemisphäre die wirtschaftlich wichtigste Öl- und Eiweisspflanze. Genutzt werden in erster Linie die Samen, die bis zu $45 \%$ Rohfett enthalten, wovon $80-90 \%$ als Öl gewonnen werden kann, welches als Speiseöl in den Handel kommt. Als Nebenprodukte fallen ein geringwertiges Stroh, sowie die als 'Ölkuchen' gehandelten Pressrückstände als wertvolles, da eiweissreiches Kraftfutter an [1]. Der Anbau von Raps hat in letzter Zeit weltweit an Bedeutung gewonnen. So wurde die Produktion von Rapsöl in den letzten 18 Jahren ungefähr verdreifacht [2]. Dies ist auch insbesondere den Züchtungserfolgen von 00 -Sorten (erucasäurefrei, d.h. max. $2 \%$ Erucasäure im Gesamtfettsäuregehalt des
Samenöls und glucosinolatarm, d.h. max. 30 $\mu$ mol Glucosinolate je g entfettetes Samenmehl) zu verdanken.

Raps gehört wie Rübsen, alle Kohlarten, brauner Senf usw. in die Familie der Kreuzblütler (Brassicaceae) und ist dic Stammform der Kohlrübe [3]. Der im Frühjahr beim Blühen stark verzweigte Hauptstengel trägt grosse, intensiv gelbe Blütentrauben, aus denen sich die zweifächrigen Schoten mit zahlreichen Samen entwickeln. Dieser Hauptstengel ist sehr regenerationskräftig. Die Blütezeit erstreckt sich über mehrere Wochen, wobei ein nur sehr kleiner Teil der Blütenanlagen zur Schotenbildung kommt. Blühende Rapsfelder sind auch beliebte Bienenweiden.

Generell sind für die gelbe bis rote Farbe von Blütenblättern neben Flavonolglykosiden (z.B. Quercetin und Kämpferol), Betaxanthine (z.B. Indicaxanthin) und Anthocyanine sehr oft Carotinoide verantwortlich, beispielsweise in Narzissen 14$]$ und Rosen. Die Farbstoffe von Rosenbluiten

\footnotetext{
*Korrespondenz: Prof. Dr. H. Pfander
} Institul für organische Chemie Universität Bern

Freiestrasse 3, $\mathrm{CH}-3012$ Bern 
I $\mathrm{H}-\left(300 \mathrm{MHz}, \mathrm{CDCl}_{3}\right)$ und ${ }^{13} \mathrm{C}-\mathrm{NMR}\left(75 \mathrm{MHz}, \mathrm{CDCl}_{3}\right)$ : vgl. Tabelle.

Generierung und Abfangen von Tricyclo[3.2.1.02,4]octa-2(4),6-dien (12). In einem ausgeheizten 25-ml-Dreihals-Rundkolben mit Septum, Thermometer und Inertgas-Überleitung werden 200 $\mathrm{mg}(0,76 \mathrm{mmol}) \mathbf{1 5} \mathrm{a}$ und $205 \mathrm{mg}(0,76 \mathrm{mmol})$ DPIBF in $5 \mathrm{ml} \mathrm{THF}$ gelöst und während 15 min mittels Spritze bei $-80^{\circ}$ bis $-75^{\circ} 0,53 \mathrm{ml}$ einer $1,4 \mathrm{M} \quad t$-BuLi Lsg. in Pentan $(0,74 \mathrm{mmol})$ zugetropft. Nach jedem Tropfen verfärbt sich die gelbe Lsg. braun. Die gelbbraune Lsg. wird während $30 \mathrm{~min}$ bei $-50^{\circ}$, dann $1 \mathrm{~h}$ bei RT. gerührt. Zur Elimination von evtl, überschüssigem DPIBF wird mit ca. $200 \mathrm{mg}$ (ca. 2,0 mmol) Maleinsäure-anhydrid versetzt und $15 \mathrm{~min}$ weitergerührt. Das Gemisch wird mil $50 \mathrm{ml} \mathrm{Et} 2 \mathrm{O}$ verdünnt, im Scheidetrichter $2 \times$ mit $2 \mathrm{~N}$ $\mathrm{NaOH}, 1 \times$ mit $\mathrm{H}_{2} \mathrm{O}$ und $\mathrm{I} x$ mit ges. $\mathrm{NaCl}$-Lsg. gewaschen, getrocknet $\left(\mathrm{MgSO}_{4}\right)$ und i.RV, bei RT. einrotiert. Das ölige Rohprodukt wird in 2 Teilen durch präp. Schichtchromatographie gereinigt (Merck Art 5717 PSC-Fertigplatten Kieselgel $60 F_{254}$, Schichtdicke: $2 \mathrm{~mm}$, Elutionsmittel Pentan/ $\mathrm{Et}_{2} \mathrm{O}$ 20:1, zweifache Elution). Die Zone mit einem $R_{\mathrm{f}}$-Wert von 0,61 wird gesammelt und mit $\mathrm{E}_{2} \mathrm{O}$ extrahiert, das Eluat i.RV bei RT. eingedampft: $119,8 \mathrm{mg}(42 \%)$ weisse Kristalle von 16, die laut lH-NMR nur Spuren von Verunrcinigungen enthalten. Schmp. $172-173^{\circ}$ (Pentan). ${ }^{1} \mathrm{H}-$ $\left(300 \mathrm{MHz}, \mathrm{CDCl}_{3}\right)$ und ${ }^{13} \mathrm{C}-\mathrm{NMR}\left(75 \mathrm{MHz}, \mathrm{CDCl}_{3}\right)$ : vgl. Tabelle. MS: $374\left(81, M^{+}\right), 307(100)$

2-Bromo-4-(trimethylsilyl)-endo-tricyclo[3.2.1.02.4 ]oci-6-en (15b). In einem ausgeheizten 25ml-Dreihals-Rundkolben mit Septum, Thermometer und Inertgas-Überleitung wird eine Lsg. von $500 \mathrm{mg}$ (1.42 mmol) 1,1,2-Tribromo-2-(trimethylsilyl)cyclopropan (13) [ 10 ] in $5 \mathrm{ml} \mathrm{THF}$ auf $-80^{\circ}$ abgekühlt. Man tropft mittels Spritze während $15 \mathrm{~min} 1,01 \mathrm{ml}$ einer 1,4M /-BuLi Lsg. in Pentan (1,4l mmol) zu. Dabei tritt eine deutliche Farbveränderung von violett über grüngelb bis braun ein. Innerhalb von $30 \mathrm{~min}$ wird unter Rühren von $-80^{\circ}$ auf $-50^{\circ}$, sodann innert $10 \mathrm{~min}$ von $-50^{\circ}$ auf RT. erwirmt und rasch mit ciner Lsg. von $0,116 \mathrm{ml}(93 \mathrm{mg}, 1,41 \mathrm{mmol})$ Cyclopentadien in $\mathrm{I} \mathrm{ml}$
THF versetzt. Das braune Gemisch wird weitere $4,5 \mathrm{~h}$ bei RT. gerührt, mit $50 \mathrm{ml} \mathrm{Et}_{2} \mathrm{O}$ verdünnt, im Scheidetrichter mit $\mathrm{H}_{2} \mathrm{O}$ und ges. $\mathrm{NaCl}-\mathrm{Lsg}$. gewaschen, getrocknet $\left(\mathrm{MgSO}_{4}\right)$ und i. RV. bei $-20^{\circ} / 0,2$ Torr eingeengt: $253,7 \mathrm{mg}$ (69\%) NMR-reines Rohprodukt $15 \mathrm{~b}$ als gelbliches Öl. Chromatographie über $15 \mathrm{~g}$ Kieselgel mit Pentan liefert $166,8 \mathrm{mg}(46 \%)$ 15b als farblose Flüssigkeit. ${ }^{1} \mathrm{H}-\left(300 \mathrm{MHz}, \mathrm{CDCl}_{3}\right)$ und ${ }^{13} \mathrm{C}$ NMR (75 $\left.\mathrm{MHz}_{2} \mathrm{CDCl}_{3}\right)$ : vgl. Tabelle.

\section{Eingegangen am 30 . November 1990}

[1] Teil der geplanten Dissertation von M.M., Universität Bern.

[2] W.E. Billups, M.M. Haley, G. A. Lee, Chem, Rev $1989,89,1147$; B. Halton, M.G. Banwell, 'Cyclopropenes', in 'The Chemistry of the Cyclopropyl Group', Ed. Z. Rappoport, Wiley, New York, 1987

[3] H.-U. Wagner, G. Szeimies, J. Chandrasekhar, P.v.R. Schleyer, J.A. Pople, J.S. Binkley, J. Am. Chem. Soc. 1978, 100, 1210.

[4] G. Szeimies, J. Harnisch, 0. Baumgärtel, J. Am. Chem. Soc. 1977, 99, 5183; U. Szeinies-Seebach, G. Szeimies, ihid. 1978, 100, 3966; U. Szeimies-Seebach, A. Schöffer, R. Römer, G. Szeimies, Chem. Ber. 1981, 114, 1767; H.-G. Zoch, G. Szeimies, R. Römer, G. Germain, J.-P. Declercq, ibid. 1983, //6, 2285.

[5] A. Weber, M. Neuenschwander, Angew. Chem. 1981, 93, 788; ibid. Int. Ed. 1981, 20, 774; A. Weber, U. Stämpfli, M. Neuenschwander, Helv. Chim. Acta 1989, 72, 29.

[6] W.E. Billups, L.-J. Lin, E.W. Casserley, J. Am. Chem. Soc. 1984, 106, 3698; S.W. Staley, T.D. Norden, ibid. 1984, 106, 3699.

[7] K.B. Wiberg, G. Bonneville, Tetrahedron Lett. $1982,23,5385$.

[8] W.E. Billups, L.-J . Lin, Tetrahedron 1986, 42 , 1575.

[9] P.J.Chenier, D.A. Southard, J.Org. Chem. 1989, 54, 3519; ibid. 1990, 55, 4333 .
[10] B.R. Dent, B. Halton, A.M.F. Smith. Aust, J. Chem. 1986, 39, 1621.

[11] In Abwesenheit von Cyclopentadien liisst sich 1,2-Dibromocyclopropen (14a) NMRspektroskopisch nachweisen: Das charakteristische Singulett der $\mathrm{CH}_{2}$-Gruppe bei $2,10 \mathrm{ppm}$ $\left(\mathrm{CDCl}_{3}\right)$ verschwindet bei Zugabe von Cyclopentadien.

[12] St.Walther, Dissertation, Universitit Bern, 1985.

[13] Während $C(8)$ von endo-Tricyclol.3.2.1.0 $0^{2.4} \mathrm{loct}-$ 6 -en bei $63.7 \mathrm{ppm}$ absorbiert. liegt $\mathrm{C}(8)$ des e'voIsomers bei $37,4 \mathrm{ppm}$ ! H. Günther, W. Herrig, $H$. Seel, S. Tobias, A de Meijere, B. Schrader, I.Org. Chen. 1980, 45, 4329; K. Tori, M. Ueyama, T. Tsuji, H. Matsumura, H. Tanida, H. I wamura, K. Kushida, T, Nishida, S. Satoh, Tetrahedron Le'll. 1974, 327.

[14] Die Abfangreaktion von 12 ist nur zu $40 \%$ effizient, die Struktur möglicher Nebenprodukte noch unbekannt. Als Folgereaktion von 12 ist vor allem dic Drciringöffnung zu einem Vinylcarben attraktiv, welches sich durch Umlagerung stabilisieren kinn. Vgl. z.B. P. Miiller, N. Pautex, Hels. Chim. Acta 1988, 71, 1630; N. Piutex, Dissertation, Universitiat Genf, 1989.

[15] Trotz dieser uberzeugenden Argumente soll versucht werden, die Struktur von 16 durch eine Röntgenstrukturanalyse zu bestïtigen.

[16] Sowohl die Signallage als auch die Kopplungskonstante $J(3 a, 3 b)$ von 16 sind den Werten analoger syn-konfigurierter Addukte von Diphenylisobenzofuran an Cyclopropene sehr ähnlich. Vgl. P. Müller, G. Bernardinelli, J. Pfyffer, D. Rodriguez, J.-P. Schaller, Holl, Chim. Acta 1988, 71, 544

[17] Aufgrund von Vorversuchen verläufı der (Halogen-Li)-Austausch sogar schneller als dic Deprotonierung von Cyclopentadien, so dass das Cyclopropan 13 mit $t$-BuLi sogar in Gegenwart von Cyclopentadien umgesetzi werden kann!

[18] W.E. Billups, M.M. Haley, Angen: Chem. 1989, 101, 1735; ibid. Int. Ed. 1989, 28, 1711.
Chimia 45 (1991) 27-29

(c) Schweiz. Chemiker-Verband; ISSN 0009-4293

\section{Isolierung und Charakterisierung der Hauptcarotinoide aus Blütenblättern von Brassica napus}

\section{Bertold Debrunner und Hanspeter Pfander*}

Abstract. From the yellow petals of rape (Brassica napus), five major carotenoids have been isolated. Based on UV/VIS, CD, 400-MHz' $\mathrm{H}-\mathrm{NMR}$, and mass spectra, they were identified as $\left(9^{\prime} Z\right)$-neoxanthin, (all-E)- and (9Z)-violaxanthin, and $\left(8^{\prime} R\right)$ - and ( $\left.8^{\prime} S\right)$-luteoxanthin.

\section{Einleitung}

Raps (Brassicanapus) ist im gemässigten Klimabereich der nördlichen Hemisphäre die wirtschaftlich wichtigste Öl- und Eiweisspflanze. Genutzt werden in erster Linie die Samen, die bis zu $45 \%$ Rohfett enthalten, wovon $80-90 \%$ als Öl gewonnen werden kann, welches als Speiseöl in den Handel kommt. Als Nebenprodukte fallen ein geringwertiges Stroh, sowie die als 'Ölkuchen' gehandelten Pressrückstände als wertvolles, da eiweissreiches Kraftfutter an [1]. Der Anbau von Raps hat in letzter Zeit weltweit an Bedeutung gewonnen. So wurde die Produktion von Rapsöl in den letzten 18 Jahren ungefähr verdreifacht [2]. Dies ist auch insbesondere den Züchtungserfolgen von 00 -Sorten (erucasäurefrei, d.h. max. $2 \%$ Erucasäure im Gesamtfettsäuregehalt des
Samenöls und glucosinolatarm, d.h. max. 30 $\mu$ mol Glucosinolate je g entfettetes Samenmehl) zu verdanken.

Raps gehört wie Rübsen, alle Kohlarten, brauner Senf usw. in die Familie der Kreuzblütler (Brassicaceae) und ist dic Stammform der Kohlrübe [3]. Der im Frühjahr beim Blühen stark verzweigte Hauptstengel trägt grosse, intensiv gelbe Blütentrauben, aus denen sich die zweifächrigen Schoten mit zahlreichen Samen entwickeln. Dieser Hauptstengel ist sehr regenerationskräftig. Die Blütezeit erstreckt sich über mehrere Wochen, wobei ein nur sehr kleiner Teil der Blütenanlagen zur Schotenbildung kommt. Blühende Rapsfelder sind auch beliebte Bienenweiden.

Generell sind für die gelbe bis rote Farbe von Blütenblättern neben Flavonolglykosiden (z.B. Quercetin und Kämpferol), Betaxanthine (z.B. Indicaxanthin) und Anthocyanine sehr oft Carotinoide verantwortlich, beispielsweise in Narzissen 14$]$ und Rosen. Die Farbstoffe von Rosenbluiten

\footnotetext{
*Korrespondenz: Prof. Dr. H. Pfander
} Institul für organische Chemie Universität Bern

Freiestrasse 3, $\mathrm{CH}-3012$ Bern 


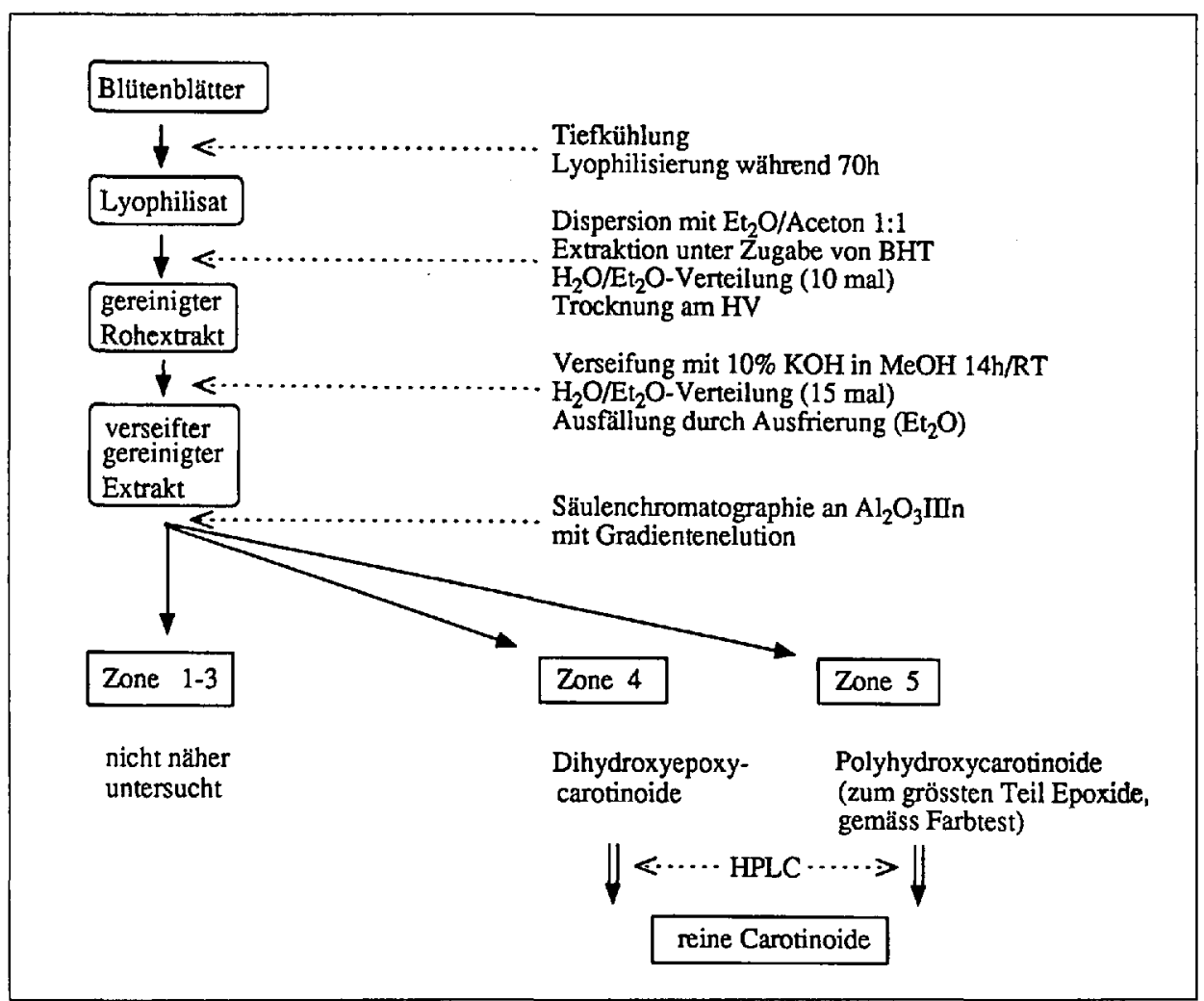

Fig. 1. Isolierschema

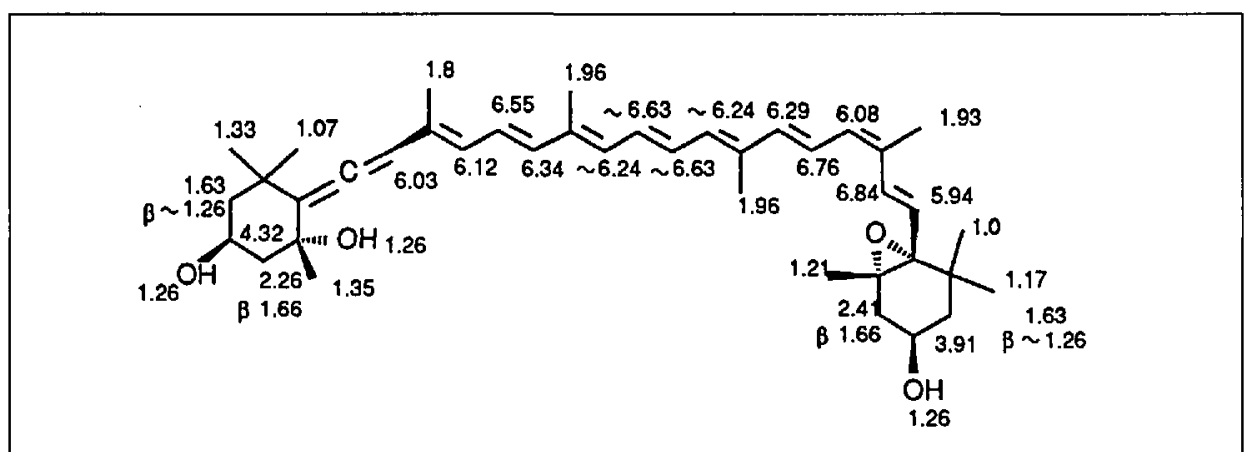

Fig. 2. ${ }^{\text {H }}$-NMR-Daten (400-MHz, $\mathrm{CDCl}_{3}$ ) von (9Z)-Neoxanthin

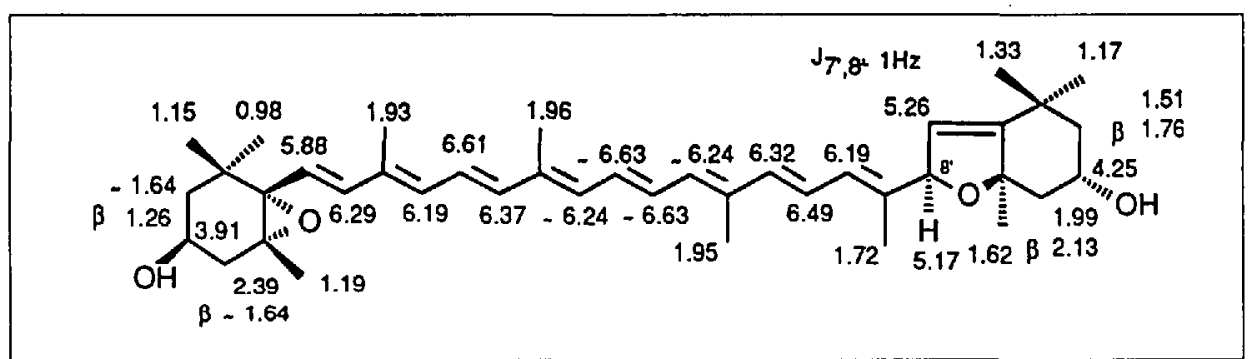

Fig. 3. ' $H$-NMR-Daten (400-MHz, $\left.\mathrm{CDCl}_{3}\right)$ von (8'R)-Luteoxanthin

sind eingehend von Eugster und Mitarbeitern untersucht worden [5-8]. So wurden aus den gelben Petalen von Rosa foetida HeRrm. 35 verschiedene Carotinoide isoliert und ihre Struktur bestimmt [9]. Auffallend ist dabei der hohe Gehalt an Carotinoidepoxiden, der ca. $87 \%$ beträgt. Der Hauptteil dieser Verbindungen ist auf Antheraxanthin und Violaxanthin zurückzuführen, was auf das Vorliegen eines defekten Violaxanthin-Zyklus hinweist. In neueren Arbeiten [10-13] ist zudem auch die Isolierung von verschiedenen Apo- und Diapocarotinoiden beschrieben worden.

In der vorliegenden Arbeit berichten wir über die Hauptcarotinoide aus den Petalen von Winterraps [14].

\section{Resultate und Diskussion}

Aus $68,5 \mathrm{~g}$ getrockneten Blütenblätter der Sorte 'Bienvenue' liessen sich nach Extraktion, Verseifung und Vortrennung des Rohextraktes an $\mathrm{Al}_{2} \mathrm{O}_{3} \mathrm{III}_{n}$ mit Gradientenelution total $626 \mathrm{mg}$ etherlösliche Carotinoide in 5 Hauptfraktionen gewinnen (s. Fig. 1 und Tab. I). Auffallend ist, in Analogie zu den Rosenblüten, der hohe Anteil von Di- resp. Polyhydroxy-epoxiden;

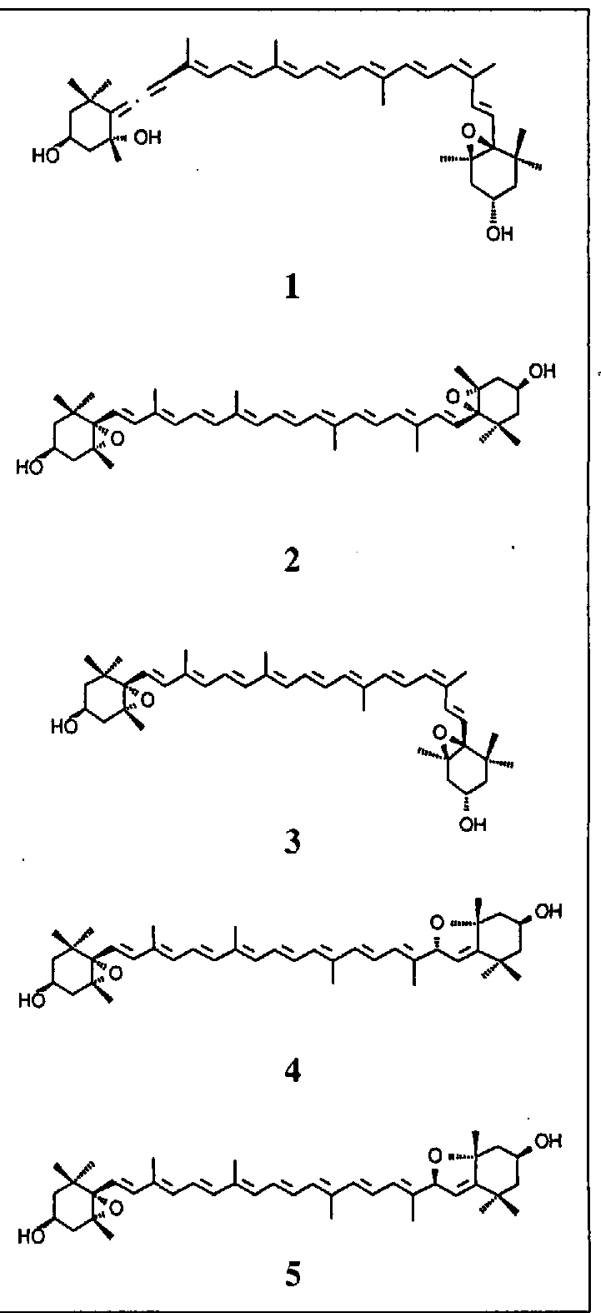

Fr. 4 und 5 (grösstenteils Epoxide) ergaben über $90 \%$ des Gesamtcarotinoidgehaltes.

Zur Strukturbestimmung wurden im folgenden aus diesen beiden Fraktionen fünf Hauptkomponenten mittels halbpräp. HPLC isoliert (s. Exper. Teil) und mit Hilfe von UV/VIS-, CD-, 400-MHz- ${ }^{-1} \mathrm{H}-\mathrm{NMR}$ - und Massenspektren eindeutig identifiziert. Auf Grund der spektroskopischen Daten können diesen Verbindungen die im folgenden angegebenen Strukturen zugeordnet werden. Aus der Zone 5 wurde $\left(9^{\prime} Z\right)$-Neoxanthin $\left(\left(9^{\prime} Z, 3 S, 5 R, 6 R, 3^{\prime} S, 5^{\prime} R, 6^{\prime} S\right)-5^{\prime}, 6^{\prime}-\right.$-Epoxy-6,7didehydro- $5,6,5^{\prime}, 6$ '-tetrahydro- $\beta, \beta$-carotin$3,5,3^{\prime}$-triol, 1$)$ isoliert, während aus Zone 4 (all-E)-Violaxanthin ((all-E,3S,$\left.5 R, 6 S, 3^{\prime} S, 5^{\prime} R, 6^{\prime} S\right)-5,6,5^{\prime}, 6^{\prime}$-Diepoxy$5,6,5^{\prime}, 6^{\prime}$-tetrahydro- $\beta, \beta$-carotin-3,3'-diol, 2 ), (9Z)-Violaxanthin $\left(\left(9 Z, 3 S, 5 R, 6 S, 3 ' S, 5^{\prime} R\right.\right.$, 6'S)-5,6,5',6'-Diepoxy-5,6,5',6'-tetrahydro$\beta, \beta$-carotin-3,3'-diol, 3), (8'R)-Luteoxanthin $\left(\left(3 S, 5 R, 6 S, 3^{\prime} S, 5^{\prime} R, 8^{\prime} R\right)-5,6,5^{\prime}, 8^{\prime}\right.$-Diepoxy$5,6,5^{\prime}, 8^{\prime}$-tetrahydro- $\beta, \beta$-carotin-3,3'-diol, 4) und $\left(8^{\prime} S\right)$-Luteoxanthin $\left(\left(3 S, 5 R, 6 S, 3^{\prime} S, 5^{\prime} R\right.\right.$,$\left.8^{\prime} S\right)-5,6,5^{\prime}, 8^{\prime}$-Diepoxy-5,6,5', ' $^{\prime}$-tetrahydro$\beta, \beta$-carotin-3,3'-diol, 5) isoliert wurde.

Die aus den Petalen von Raps bisher isolierten Carotinoide sind bereits früher in verschiedenen anderen Organismen gefunden worden. Neben (9Z)-Neoxanthin (1) konnte auch das (all-E)-Isomere nachgewiesen werden, doch konnte dieses nicht in völlig reiner Form isoliert werden; mengenmässig überwog bemerkenswerterweise das (Z)-Isomere. Die Daten der 400- 
Tabelle. Menge und Zusammensetzung der Carotinoide aus B.napus nach Trennung an $\mathrm{Al}_{2} \mathrm{O}_{3} \mathrm{III}_{n}$ a)

\begin{tabular}{|c|c|c|c|}
\hline \multicolumn{2}{|c|}{ Zone } & Menge $[\mathrm{mg}]$ & \multirow{2}{*}{$\frac{\text { in } \%}{2,9}$} \\
\hline 1 & Kohlenwasserstoffe & 18 & \\
\hline 2 & Caroniodaldehyde und -keto & 20 & 3,1 \\
\hline 3 & Mono- und Dihydroxycaroti & le 18 & 2,8 \\
\hline 4 & Dihydroxyepoxycarotinoide & 496 & 79,2 \\
\hline \multirow[t]{2}{*}{5} & Polyhydroxycarotinoide & 75 & 12,0 \\
\hline & Total & 627 & \\
\hline
\end{tabular}

a) Die aus UV/VIS-Messungen abgeschätzten Gehaltsan-
gaben beziehen sich auf ô g des verseiften Extraktes.

MHz- ${ }^{-}$H-NMR-Spektren von 1 sind mit denjenigen welche kürzlich von $M a ̈ r k i-F i$ scher und Eugster [15] veröffentlicht wurden in Einklang und sind in Fig. 2 gezeigt. (all-E)-Violaxanthin 2, als auch dessen (9Z)Isomeres 3 wurden früher aus $R$. foetida isoliert [8], ebenfalls die beiden Luteoxanthine 4 (400- $\mathrm{MHz}^{-}{ }^{1} \mathrm{H}-\mathrm{NMR}-\mathrm{Daten} \mathrm{s}$. Fig. 3) und 5.

Neben der Strukturaufklärung der Hauptcarotinoide wurden die Resultate unserer Voruntersuchungen insofern bestätigt, dass eine grosse Anzahl von verschiedenen Carotinoiden in den Blütenblättern von Raps vorhanden ist. Nach einer DC-Analyse konnten im verseiften Gesamtextrakt 20 Komponenten festgestellt werden. Die HPLC-Analyse jedoch zeigte, dass das Gesamtcarotinoidspektrum wesentlich vielfältiger ist. So kann die Anzahl der Carotinoide in den gelben Blütenblättern auf über 50 geschätzt werden. Die Arbeiten zur Strukturaufklärung der weiteren Carotinoide, sowie deren quantitative Zusammensetzung sind zur Zeit im Gang.

\section{Experimentelles}

I. Allgemeines. Sämtliche Operationen wurden unter Ar und unter Lichtausschluss durchgeführt. Die verwendeten Lsgm. waren von p.a.-Qualität oder wurden nach den üblichen Methoden [8] vorgereinig und vor Gebrauch frisch destilliert. Das Einengen der Lsgm. am RV. erfolgte bei $25^{\circ}$. Die Gehaltsbestimmung für Gemische oder für Carotinoide mit unbekannter quantitativer Extinktion wurde aus $\mathrm{E}_{\mathrm{c} / \mathrm{t}}^{\mathrm{t}}$-Werten mit 2500 als Standard am Hauptmaximum berechnet. HPLC HPLC-Analysen erfolgten analytisch $(20 \mu \mathrm{m})$ an Spherisorb 5 ODS-2 $(4,6 \times 250 \mathrm{~mm})$ oder semipräp. $(100 \mu \mathrm{m})$ an Nucleosil $5 C_{18}(10 \times 250 \mathrm{~mm})$, (Macherey Nagel, Düren, BRD). Pumpen Altex 110 A mit Mikroprozessor Altex 420. Aufnahme der Chromatogramme und der UV/VIS-Spektren mit Kontron Uvikon LCD 725-Detektor und SpektrophotometerPerkin-Elmer 554 oder Photodiodenarray Waters 990 Detector mit NEC $A P C$ IV-Computer (Power Mate 2), NEC Pinwriter CP 6 und Waters 990 Plotter. Angabe von $\lambda_{\max }$ in $\mathrm{nm}$. Alle Lsgm. (Romil Chemicals, Kölliken) für die Chrom:tographie waren HPLC-Qualität und wurden vor Gebrauch durch Glass-Microfibre-Filter $G F / F$ (Whatman, Maidstone, GB, $\phi 2,5 \mathrm{~cm}$ ) gefiltert. Als Spritzenfilter dienten Alltech HPLC-Filter der Serie 6.000 (Nylon 66 $(0,45 \mu))$. CD-Spektren: Modifizierter Dichrograph II (Jobin-Yvon), Angaben in $\mathrm{nm}(\Delta \varepsilon)$ aufgenommen bei RT. in EtOH. ${ }^{1} \mathrm{H}-\mathrm{NMR}$-Spektren: 400 MHz Bruker Spektrospin AM 400 WB mit Dual-Kopf, Lsgm. $\mathrm{CDCl}_{3} . \delta$ in ppm bezüglich TMS $(=0 \mathrm{ppm}) . J$ in Hz. MS: MS 9 ( $A E$, Manchester, GB) mit ZAB-Konsol
(VG, Altrincham, GB). Datensystem SS 300 (Finnigan $M A T$, Bremen, BRD). Probenzuführung auf der Spitze eines Glasstäbchens $(\phi \quad 1 \mathrm{~mm})$. Im $\mathrm{N}_{2}$-Strom zur Trockene eingedampft und über eine Vakuumschleuse direkt in der Ionenquelle bei $\sim 260^{\circ}$ verdampft. lonisierungsenergie 70 eV. DC: Fertigplatten, Kieselgel $60 F_{254}\left(20 \times 20 \mathrm{~cm}\right.$, Merck). SC: $\mathrm{Al}_{2} \mathrm{O}_{3}$ (I neutral, Camag, Muttenz). $\mathrm{Al}_{2} \mathrm{O}_{3}$ (III neutral) wurde durch Zugabe von $6 \% \mathrm{H}_{2} \mathrm{O}$ zu Al $\mathrm{Al}_{2} \mathrm{O}_{3}$ (I neutral) und $12 \mathrm{~h}$ Rühren erhalten. Rapsbluitenblätter der Sorte 'Bienvenue' wurden zu Beginn der Blütezeit anfangs Mai 1988 während zweier Wochen bei H. Huber in Kiesen/BE zusammengetragen.

2. Ausgangsmaterial und Extraktion. Total $1400 \mathrm{~g}$ Blütenblätter von Brassica napus wurden sofort nach dem Pflücken bei $-18^{\circ}$ tiefgefroren, dann portionenweise in einem flachen, grossen Dewar mit flüssigem $\mathrm{N}_{2}$ ausgefroren, gemörsert, in Lyophilisierungskolben gefüllt und mehrere Tage an der Gefriertrocknungsanlage belassen. Bis zur weiteren Verwendung wurde das so aufgeschlossene Pflanzenmaterial $(200 \mathrm{~g}$ ) im Tiefkühlfach gelagert. Die getrocknete Petalen (68,5 g) wurden in ein Aceton/Et2 $\mathrm{O}$ ।:1 Gemisch eingelegt und mit einem Hochleistungs-Dispergiergerät (Polytronic) zu einem feinen Brei zerkleinert und mehrmals mit frischen Portionen Lsgm. extrahiert. Dies ergab eine quantitative Extraktion der Farbstoffe (Ruickstand annähernd farblos). Die gelbe Lsg. wurde nach jedem Extraktionsschritt sofort am $R V$. eingeengt. Der honigartige Extrakt wurde dann zwischen $\mathrm{H}_{2} \mathrm{O}$ und $\mathrm{Et}_{2} \mathrm{O}$ verteilt und die aus der $\mathrm{Et}_{2} \mathrm{O}$-Phase gewonnenen Anteile $(16 \mathrm{~g}) \mathrm{mit} 300 \mathrm{ml} 10 \% \mathrm{KOH} / \mathrm{MeOH}$ bei RT während $14 \mathrm{~h}$ verseift und erneut zwischen $\mathrm{H}_{2} \mathrm{O}$ und $\mathrm{Et}_{2} \mathrm{O}$ verteilt. Durch Ausfrieren bei-1 $18^{\circ}$ konnten, nach Filtration über Celite, aus dem etherlöslichen Extrak farblose Substanzen entfernt werden.

3. Säulenchromatographie. Die Grobtrennung in 5 Zonen erfolgte mittels Gradientelution mit je 2,5 g Extrakt an $\mathrm{Al}_{2} \mathrm{O}_{3} \mathrm{III} n$ ( 500 g. Säule: $\phi 5 \times 30 \mathrm{~cm}$, nass gefüllt) in folgender Reihenfolge: Petrolether/Toluo $\mathrm{I}: 1 \rightarrow$ Toluol $/ \mathrm{Et}_{2} \mathrm{O} 3: 1 \rightarrow \mathrm{Et}_{2} \mathrm{O} \rightarrow \mathrm{AcOEt} \rightarrow \mathrm{MeOH}$. Der Extrakt wurde in Petrolether/Toluol $1: 1$ aufgezogen und die Elution erfolgte mit steigender Polarität Der Wechsel der Eluiermittel geschah jeweilen erst, nachdem eine Zone gesammelt wurde. Die resultierenden Mengen der fünf gelben Zonen nach dem Einengen am RV. und der Trocknung am $\mathrm{HV}$. sind in der Tabelle ersichtlich.

4. HPLC. Die semipräp. HPLC-Trennung der Zone 5 geschah mit Eluent $1: 15 \% \mathrm{~B}$ in A+B, $3 \mathrm{ml} / \mathrm{min}$ Fluss, wobei $\mathrm{A}: 90 \mathrm{~T} \mathrm{MeOH}, 10 \mathrm{~T} \mathrm{H}_{2} \mathrm{O}, 1 \mathrm{~T} \mathrm{Et} 3 \mathrm{~N}$ und $\mathrm{B}: 20$ $\mathrm{T}$ AcOEt, $80 \mathrm{~T} \mathrm{MeOH}, 1 \mathrm{~T} \mathrm{Et}_{3} \mathrm{~N}$. Die Retentionszeiten waren für (all-E)-Neoxanthin: $12,5 \mathrm{~min}$, für $\left(9^{\prime} Z\right)$ Neoxanthin: 14 min, womit eine Grundlinientrennung gelang und beide Carotinoide einzeln aufgefangen werden konnten. Die Trennung der Zone 4 geschah mit Eluent 2: $80 \% \mathrm{C}$ in $\mathrm{A}+\mathrm{C}, 2,3 \mathrm{ml} / \mathrm{min}$ Fluss, wobei $\mathrm{C}$ MeCN. Die Retentionszeitenwaren für (all-E) Violaxanthin: $11,7 \mathrm{~min}$, für $\left(8^{\prime} R\right)$-Luteoxanthin: $12,5 \mathrm{~min}$ und für $(8 ' S)$-Luteoxanthin: $13,1 \mathrm{~min}$. Für (all-E) Violaxanthin gelang damit eine Grundlinientrennung und wurde rein aufgefangen. Die Trennung von (8'S) Luteoxanthin und einem $\left(8^{\prime} R\right)$-Luteoxanthin/ $(9 Z)$ Violaxanthin-Gemisch mit Eluent 3: 97\% D in C+D $2,3 \mathrm{ml} / \mathrm{min}$ Fluss, wobei: D: $90 \mathrm{~T} \mathrm{i}-\mathrm{PrOH}, 10 \mathrm{~T} \mathrm{H}_{2} \mathrm{O}$, T Et ${ }_{3} N$. $t_{R}$ für das $\left(8^{\prime} R\right)$-Luteoxanthin $/(9 Z)$ Violaxanthin-Gemisch: 17,3 min und für ( 8 'S)-Luteoxanthin: $18,3 \mathrm{~min}$, womit cine Grundlinientrennung gelang und $\left(8^{\prime} S\right)$-Luteoxanthin aufgefangen wurde. Die Trennung von $\left(8^{\prime} R\right)$-Luteoxanthin und $(9 Z)$ Violaxanthin gelang mit $30 \% \mathrm{~B}$ in $\mathrm{A}+\mathrm{B}, 2,5 \mathrm{ml} / \mathrm{min}$ Fluss. $t_{\mathrm{R}}$ für $\left(8^{\prime} R\right)$-Luteoxanthin: $17,3 \mathrm{~min}$ und für $(9 Z)$ Violaxanthin: $18,2 \mathrm{~min}$, womit eine Grundlinientrennung gelang und beide Carotinoide cinzeln gesammelt werden konnten. Die Reinheit der einzeln aufgefangenen Komponenten wurde zusätzlich durch wiederholtes Auftragen auf die Säule mit unterschiedlichen Fliessmittel bestätigt.

5. Charakterisierung der Carotinoide. 5.1. (9Z) Neoxanthin (1). UV/VIS: 464, 435, 412, 327 (Hexan) $\alpha / \beta=0,83$. CD: 471,3 (11,59), 265,5 (4,87), 256,6 $(3,45), 227,8(-7,80), 204,3(-3,30)$. 1 H-NMR $(400$ $\left.\mathrm{MHz}_{1} \mathrm{CDCl}_{3}, \mathrm{Fig} .5\right)$ : Signallagen in Übereinstimmung mit [15], zusätzlich: $1,63\left(\mathrm{~m}, \mathrm{H}_{\mathrm{eq}}-\mathrm{C}\left(2 / 2^{\prime}\right)\right) ; 1,66(\mathrm{~m}$ $\left.\mathrm{H}_{\mathrm{ax}}-\mathrm{C}\left(4 / 4^{\prime}\right)\right) ; 2,26\left(m, \mathrm{H}_{\mathrm{eq}}-\mathrm{C}(4)\right) ; 2,4 \mathrm{l}\left(m, \mathrm{H}_{\mathrm{eq}}-\mathrm{C}\left(4^{\prime}\right)\right)$.
MS: $600\left(M^{+}\right), 582\left([M-18]^{+}\right), 566,542\left([M-58]^{+}\right)$, $502\left([M-18-80]^{+}\right), 221,43$

5.2. (all-E)-Violaxanthin (2). UV/VIS: 470, 440, $416(\mathrm{EtOH}), \alpha / \beta=0,83 . \mathrm{CD}: 478,7(19,38), 450$, 1 $(14,41), 421,1(11,59), 397,1(9,98), 327,4(9,79), 264,0$ $(-28,40), 227,9(17,60), 204,0(-3,21)$. 'H-NMR (400 $\mathrm{MHz}, \mathrm{CDCl}_{3}$ ): Signallagen in Übereinstimmung mit [17].

5.3. (9Z)-Violaxamthin (3). 'H-NMR (400) MHz, $\mathrm{CDCl}_{3}$ ), Signallagen in Übereinstimmung mit [9], zulsätzlich: $2,39\left(d q, \mathrm{H}_{\mathrm{eq}}-\mathrm{C}\left(4 / 4^{\prime}\right)\right) ; 6,20(d, \mathrm{H}-\mathrm{C}(10)) ; 6,25$ $\left(m, \mathrm{H}-\mathrm{C}\left(14 / 14^{\prime}\right)\right) ; 6,29\left(d, \mathrm{H}-\mathrm{C}\left(12^{\prime}\right)\right) ; 6,37(d, \mathrm{H}-\mathrm{C}(12))$; $6,60(d d, \mathrm{H}-\mathrm{C}(11)) ; 6,63\left(\mathrm{~m}, \mathrm{H}-\mathrm{C}\left(15 / 15^{\prime}\right)\right)$

5.4. (8'R)-Luteoranthin (4). UV/VIS: $448,421,398$ (Hexan); $\alpha / \beta=0,98$. CD: 462,9 (3,01), 444,6 (13,23), $415,6(11,25), 392,9(8,69), 372,7(5,83), 310.1$ $(-6,06), 298,3(-4,83), 288,3(-2,64), 267,9(-0,34)$, $243,7(4,11), 216,2(0,82)$. ' $\mathrm{H}-\mathrm{NMR}\left(400 \mathrm{MHz}, \mathrm{CDCl}_{3}\right)$, Signallagen in Übereinstimmung mit [9], zusiitzlich: $1,26\left(m, \mathrm{H}_{\mathrm{ax}}-\mathrm{C}(2)\right) ; 1,51\left(m, \mathrm{H}_{\mathrm{eq}}-\mathrm{C}\left(2^{\prime}\right)\right) ; 1,63(\mathrm{~m}$, $\left.\mathrm{H}_{\mathrm{eq}}-\mathrm{C}(2)\right) ; \mathrm{I}, 64\left(m, \mathrm{H}_{\mathrm{ax}}-\mathrm{C}(4)\right) ; \mathrm{l}, 76\left(m, \mathrm{H}_{\mathrm{ax}}-\mathrm{C}\left(2^{\prime}\right)\right) ; 1,99$ $\left(m, \mathrm{H}_{\mathrm{eq}}-\mathrm{C}\left(4^{\prime}\right)\right) ; 2,13\left(m, \mathrm{H}_{\mathrm{ax}}-\mathrm{C}(4)\right) ; 2,39\left(m, \mathrm{H}_{\mathrm{eq}}-\mathrm{C}(4)\right)$; $6,19\left(d, \mathrm{H}-\mathrm{C}\left(10^{\prime}\right)\right) ; 6,24\left(m, \mathrm{H}-\mathrm{C}\left(14 / 14^{\prime}\right)\right) ; 6,32(d$, $\left.\mathrm{H}-\mathrm{C}\left(12^{\prime}\right)\right) ; 6,37(d, \mathrm{H}-\mathrm{C}(12)) ; 6,49\left(d d, \mathrm{H}-\mathrm{C}\left(1 l^{\prime}\right)\right) ; 6,63$ $\left(m, \mathrm{H}-\mathrm{C}\left(15 / 15^{\prime}\right)\right)$. MS: $600\left(\mathrm{M}^{+}\right), 520\left([M-80)^{+}\right), 40$ $\left([M-80-80]^{+}\right), 340,299,221,181,43$.

5.5. (8'S)-Luteoxanthin (5). UV/VIS: $448,421,398$ (Hexan); $\alpha / \beta=0,98$. CD: $456,7(14,11), 429,8(8,34)$, $403,0(6,12), 386,1(5,01), 362,1(2,85), 329,8(-0,08)$, $315,2(0,76), 303,6(0,39), 249,1(-14,81), 229,7$ $(-3,93), 199,2(-12,32)$. $1 \mathrm{H}-\mathrm{NMR}\left(400 \mathrm{MH}_{2}, \mathrm{CDCl}_{3}\right)$ : Signallagen in Übereinstimmung mit [9], zusäitzlich: $1,26\left(m, \mathrm{H}_{\mathrm{ax}}-\mathrm{C}(2)\right) ; 2,12\left(m, \mathrm{H}_{\mathrm{ax}}-\mathrm{C}(4)\right) ; 2,38(\mathrm{n}$, $\left.\mathrm{H}_{\mathrm{eq}}-\mathrm{C}(4)\right) ; 6,19\left(d, \mathrm{H}-\mathrm{C}\left(10^{\prime}\right)\right) ; 6,24\left(\mathrm{~m}, \mathrm{H}-\mathrm{C}\left(14 / 14^{\prime}\right)\right)$; 6,32 (d, H-C(12)); 6,37 (d, H-C(12)); 6,51 (dd, $\left.\mathrm{H}-\mathrm{C}\left(1 \mathrm{I}^{\prime}\right)\right) ; 6,63\left(\mathrm{~m}, \mathrm{H}-\mathrm{C}\left(15 / 15^{\prime}\right)\right)$ MS: $600\left(\mathrm{M}^{+}\right), 520$ $[M-80]^{+}, 440[M-80-80]^{+}, 340,299,221,181.43$.

Wir danken dem Schueizerischen Nationalfonds und der Firma $F$. Hoffmann-La Roche $A G$, Basel für die Unterstützung dieser Arbeit.

Eingegangen am 6. Dezember 1990

[1] P. Schütt, 'Weltwirtschaftspflanzen', Paul Parey Verlag, Berlin-Hamburg, 1972, S. 94.

[2] Raps-Förderungs-Fonds (RFF), Bonn, 'Raps auf neuen Wegen', Verlag Th. Mann, GelsenkirchenBuer, 1986

[3] A. Wehrli, W. Häberli, H. Meyer, R. Weiss, W.G. Bieri, 'Ackerbau', Lindwirtschaftliche Lehrmittelzentrale, Zollikofen, 1986, S. 179.

[4] D. Berset, H. Pfander, Helv. Chim. Acta 1985,68, 1149.

[5] E.Märki-Fischer, C.H.Eugster,Helv. Chim. Ac'st $1990,73,1205$ und vorangegangene Mitteilungen

[6] C.H. Eugster, in 'Cárotenoid Chemistry and Biochemistry', Eds. G. Britton und T.W Goodwin, Pergamon Press, Oxford, 1982, S. 1.

[7] C.H. Eugster, Pure Appl. Chem. 1985, 57, 639.

[8] C.H. Eugster, in 'Carotenoids: Chemistry and Biology', Eds. N.I. Krinsky, N.M. MathewsRoth und R.F.Taylor, Plenum Press, New York, 1990, S. 1

[9] E. Märki-Fischer, R. Buchecker, C.H. Eugster, Helv. Chim. Acta 1984, 67, 2143.

[10] E.Märki-Fischer, C.H. Eugster, Helv.Chim.Acta 1987, 70, 1988

[11] E. Märki-Fischer, P. Uebelhart, C.H. Eugster, Helv. Chim. Acta 1987, 70, 1994

[12] E.Märki-Fischer, C.H. Eugster, Heh' Chim.Acta 1988, 7I, 24

[13] E. Märki-Fischer, C.H. Eugster, Helt.Chim.Actu 1988, 7I, 149l

[14] B. Debrunner, Lizentiatsarbeit, Universität Bem, 1989

[15] E. Märki-Fischer, C.H. Eugster, Hel'. Chim.Acta 1990, 73, 1637.

[16] R. Müller, R. Keese, 'Grundoperationen der' praparativen organischen Chemie', Juris Verlig, Zürich, 1981

[17] M.Acemoglu,P. Uebelhart, M. Rey,C.H. Eugster. Helv. Chim. Acta 1988, 7I, 931 . 